\title{
Ermittlung und Analyse elektro-mechanischer Belastungs- kollektive an elektronischen Komponenten in Flurförderzeugen
}

\author{
Dipl.-Wirtsch.-Ing. Stephanie Schmidt, Prof. Dr.-Ing. habil. Thorsten Schmidt \\ Technische Universität Dresden \\ Professur für Technische Logistik
}

Dipl.-Ing. Stefan Weigelt, Prof. Dr.-Ing. Ludger Overmeyer

Leibniz Universität Hannover

Institut für Transport- und Automatisierungstechnik

\begin{abstract}
Durch die rapide Zunahme der Komplexität und die besonderen Einsatz und Umgebungsbedingungen sind bei elektronischen Komponenten in Flurförderzeugen in den letzten Jahren steigende Ausfallraten zu beobachten. Daher arbeiten das Institut für Transport- und Automatisierungstechnik (ITA) der Leibniz Universität Hannover und das Institut für Technische Logistik und Arbeitssysteme (TLA) der Technischen Universität Dresden zusammen an der Entwicklung einer Prüf- und Dimensionierungsmethodik für Elektronikkomponenten in Flurförderzeugen. Nach der Auswertung bisher aufgetretener Ausfälle und der Identifikation der flurförderzeugspezifischen Einsatz- und Umgebungsbedingungen wurden Versuchsstapler mit Sensoren und Messsystemen zur Aufnahme von mechanischen Beanspruchungen sowie Strom- und Spannungsverläufen ausgestattet. Die folgenden Messungen erfolgten anhand von praxisnahen und reproduzierbaren Arbeitsspielen.
\end{abstract}

\section{$1 \quad$ Einleitung}

Flurförderzeuge werden aufgrund ihrer flexiblen Einsatzmöglichkeiten in nahezu allen Wirtschaftzweigen für das Transportieren und Umschlagen von Gütern eingesetzt. Die hohen jährlichen Aufwendungen für Wartungs- und Instandhaltungskosten von etwa 15\% der Investitionssumme nach VDI 2695 [VDI10a] schmälern jedoch die Produktivität ihrer Betreiber. Daher forschen das ITA und das TLA seit Ende $2009 \mathrm{im}$ Rahmen des über die Aif geförderten Vorhabens Dimensionierung elektronischer Komponenten an einer Methodik zur Erhöhung der Ausfallsicherheit von elektronischen Komponenten in Flurförderzeugen.

Im Fahrbetrieb sind die Elektronikkomponenten von Flurförderzeugen verschiedenen elektrischen und mechanischen Belastungsarten ausgesetzt, die sich teils deutlich von den Einwirkungen auf elektronische Bauteile in anderen Einsatzfeldern unterscheiden. Dies liegt unter anderem an der hohen Masse und der geforderten Kippstabilität, die zu relativ geringen Federwegen führen. Des Weiteren belasten die hohen Anlauf- und Rückspeiseströme, die aus den häufigen Beschleunigungs- und Bremsvorgängen resultieren, elektrisch betriebene Flurförderzeuge in besonderem Maße. Für die Zuverlässigkeit und Lebensdauer der verwendeten Elektronikkomponenten sind daher vor allem die Einflussgrößen Fahrweg und Umgebung sowie Einsatzintensität und Auslastung von Bedeutung, die auch den definierten Beanspru(c) 2010 Logistics Journal : Proceedings - ISSN 2192-9084 
chungskategorien der VDI-Richtlinie 4461 [VDI10b] zugrunde liegen.

In einer Schwachstellenanalyse wurden Bauteile der Leistungselektronik sowie Bedien- und Anzeigeelemente eines Schubmaststaplers für die weiteren Untersuchungen im Rahmen des Projekts ausgewählt.

Die während des Betriebs von Gabelstaplern auftretenden mechanischen und elektrischen Belastungen werden derzeit an den betrachteten Komponenten aufgenommen und analysiert. In den folgenden Projektschritten werden diese mit den ermittelten elektro-mechanischen Belastungskollektiven beaufschlagt, um Modelle zur Darstellung des Zusammenhangs von Belastungen und Ausfällen zu erarbeiten.

\section{Hydropulser-Messung zur Ermittlung mechanischer Belastungen}

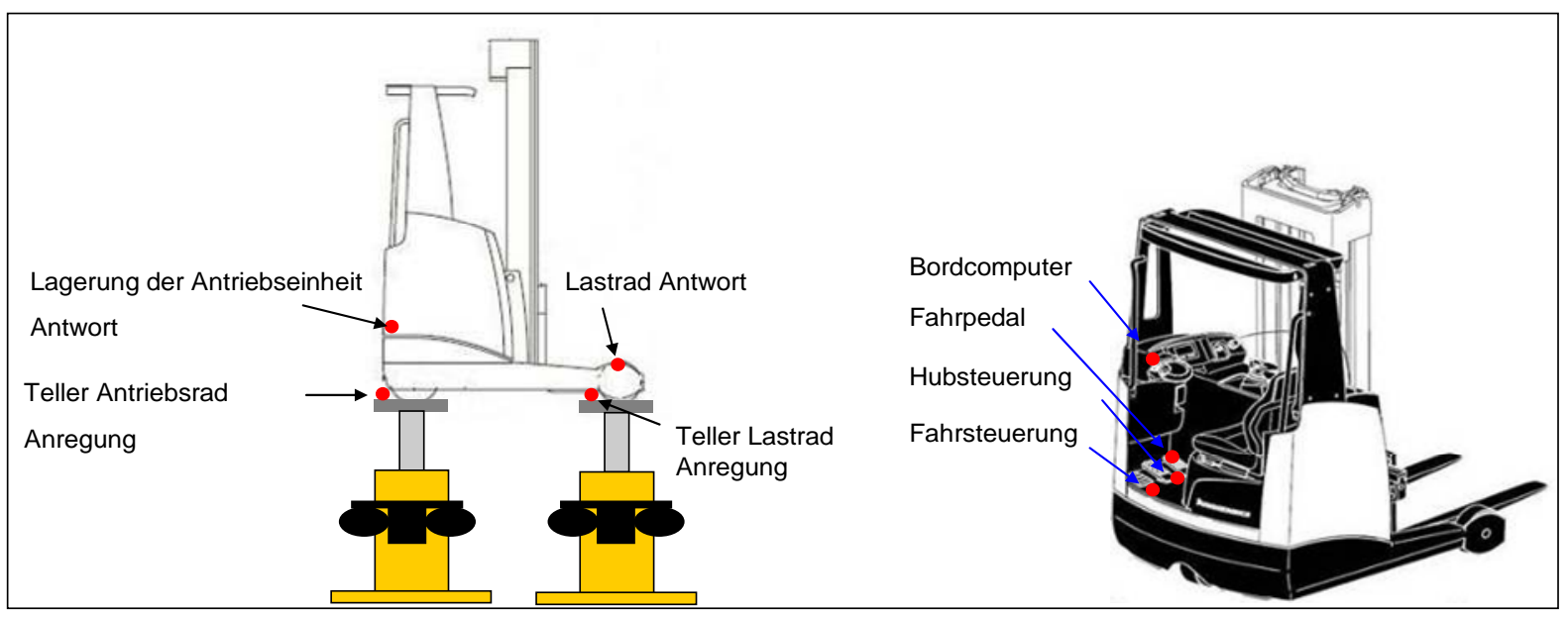

Abbildung 1: Versuchsaufbau und Lage der betrachteten Elektronikkomponenten

Zur Aufnahme der mechanischen Belastungskollektive werden die Messungen auf einem Hydropulser-Prüfstand an der TU Dresden ausgewertet. Hierbei werden die Reifen des Schubmaststaplers über hydraulisch angetriebene sogenannte Teller unabhängig voneinander angeregt. Die Frequenzverläufe werden aus den Frequenzen gebildet, die im Fahrbetrieb häufig beobachtet wurden. Die Prüfung umfasst unterschiedliche Lastfallmodule wie einen signifikanten Ausschnitt eines Kundenbodens oder eine Fahrt über eine versetzte Schwelle. Zur Bestimmung der messbaren Belastungen an den elektronischen Komponenten in Form von Beschleunigungsverläufen werden die Bauteile aus den Bereichen der Leistungselektronik und der Bedienelemente (vgl. Abbildung 1) in vertikale Richtung (z-Koordinate) und quer zur Fahrzeugachse (y-Koordinate) mit Sensoren ausgestattet. Die Fahrzeuglängsrichtung wird hierbei aufgrund der stationären Lage des Staplers auf dem Hydropulser ohne Brems- und Beschleunigungsvorgänge vernachlässigt. Da in vorausgegangenen Laborversuchen gezeigt werden konnte, dass die höchsten mechanischen Belastungen des Staplers bei einer Schwellenüberfahrt auftreten, eignet sich dieser Prüfstand dennoch insbesondere zur Untersuchung von Maximalbeanspruchungen. Diese nehmen im Rahmen der Zuverlässigkeitsprüfung eine wichtige Rolle ein. 
Die direktere Übertragung von Bodenschwingungen auf den Fahrzeugrahmen eines Schubmaststaplers im Vergleich zu einem Gegengewichtsstapler liegt insbesondere an der vergleichsweise harten Vulkollanbereifung. Diese Reifen erweisen sich bei den hohen Biege- und Torsionsmomenten, die aus dem häufigen Operieren dieser Stapler in großen Höhen resultieren, als weniger schwingungsanfällig [Bei94]. Der Werkstoff Vulkollan ${ }^{\circledR}$ weist unter anderem eine hohe Shore-Härte, die eine geringe Einfederung zur Folge hat, und eine über einen großen Temperaturbereich konstante Dämpfung auf [Bay10].

Im Zusammenhang mit Zuverlässigkeitsuntersuchungen gilt im Allgemeinen, dass ein zyklisch beanspruchtes Bauteil bereits bei wesentlich geringerer Belastung ausfällt als ein statisch beanspruchtes. Zyklische Belastungen können regelmäßige Schwingungen oder unregelmäßige Anregungen sein. Wie in Abbildung 2 dargestellt, handelt es sich bei der Hydropulser-Messung um unregelmäßige Anregungen. Diese werden insbesondere durch Stoßbelastungen hervorgerufen und bilden einen Teil der typischen Belastungen eines Flurförderzeugs im Kundenbetrieb. In Abschnitt 2.1 werden die ermittelten Schwingungskurven anhand der Zeitverläufe und mithilfe einer Fourier-Transformation analysiert. Eine Schwingungsdiagnose wird häufig auch an Antrieben von Werkzeugmaschinen zur Vermeidung von Erregerfrequenzen in der Nähe von Eigenfrequenzen von Maschinenteilen durchgeführt.

\subsection{Auswertung der Messdaten}

In einem ersten Schritt werden die gemessenen vertikalen Beschleunigungswerte der Lagerung der Antriebseinheit, der Lasträder und der oben genannten Elektronikkomponenten verglichen. Hierfür werden jeweils die maximalen positiven und negativen Beschleunigungen, ihr Durchschnittswert und ihre Standardabweichung ermittelt. Es zeigt sich, dass der Bordcomputer, der im oberen Teil der Fahrerkabine montiert ist, die höchsten durchschnittlichen Beschleunigungen erfährt, gefolgt von dem Fahrpedal. Die niedrigsten Werte werden für die Steuerungen, die sich im unteren Teil des Fahrzeugrahmens befinden, ermittelt. Außerdem liegen die maximalen Beschleunigungswerte in y- und z-Richtung bei den einzelnen Bauteilen jeweils nahe beieinander. Maximale Querbeschleunigungen werden auf dem Prüfstand bei stark gegenläufiger Bewegung der Teller unter den beiden Lasträdern beobachtet. Im Fahrbetrieb werden die höchsten Querschwingungen ebenfalls durch Bodenanregungen hervorgerufen. Eine wichtige Schwingungsursache bildet in diesem Zusammenhang die große Masse des oberen Querträgers an dem Außenmast, die zur Verminderung der Biege- und Torsionsmomente beim Hubvorgang dient. Die höheren Belastungen stellen für ein Anbringen von Sensorik und Aktorik in diesem Bereich eine besondere Herausforderung dar.

In einem nächsten Schritt werden aus den Datensätzen kurze Abschnitte ausgewählt und die Zeitverläufe betrachtet. Aus Abbildung 2 wird deutlich, dass die vertikalen Beschleunigungen der einzelnen Elektronikkomponenten auch im Zeitverlauf ähnliche Amplituden wie die Querbeschleunigungen aufweisen. Aus dem Diagramm geht 
wie aus den oben berechneten Extrem- und Durchschnittswerten hervor, dass der Bordcomputer am anfälligsten auf Bodenschwingungen reagiert, was sich in den höchsten Amplituden und niedrigsten Frequenzen (ca. $30 \mathrm{~Hz}$ ) bei geringer Dämpfung niederschlägt. Die Frequenzen der Steuerungen sind niedriger und zeigen einen relativ gleichmäßigen Verlauf. Bei entsprechenden Werkstoffen und Montagetechnik gilt im Allgemeinen, dass Bauteile, die mit geringen Frequenzen schwingen instabiler sind als Bauteile, die erst bei Anregung durch höhere Frequenzen zu schwingen beginnen.

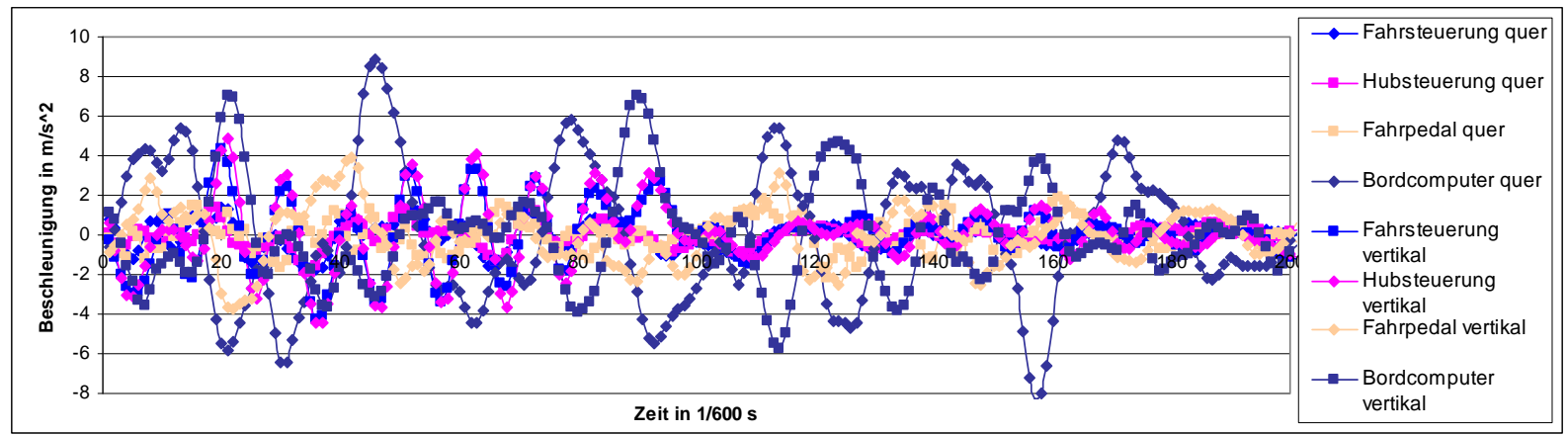

Abbildung 2: Beschleunigungssignale der oben genannten Elektronikkomponenten

Wie bereits erwähnt treten beim Einsatz von Flurförderzeugen insbesondere durch Stoßbelastungen unregelmäßige Schwingungen auf. Da die Anregung durch den Hydropulser auf einem nichtperiodischen Beschleunigungssignal beruht, fällt eine Abgrenzung und Zuordnung der Ursachen für Unregelmäßigkeiten der Schwingungen schwer. Bei dem Hydropulser-Prüfstand kommen insbesondere Stöße als Schwingungsursache in Frage. Aus der Literatur sind die Zeitverläufe beispielsweise von Spiel- und Mehrfachstößen schnell umlaufender Maschinenteile bekannt [Zsc00]. Bei detaillierter Betrachtung der Beschleunigungen in der Nähe der Umkehrpunkte der Teller (vgl. Abb. 3) zeigen sich ähnliche Schwingungsverläufe.
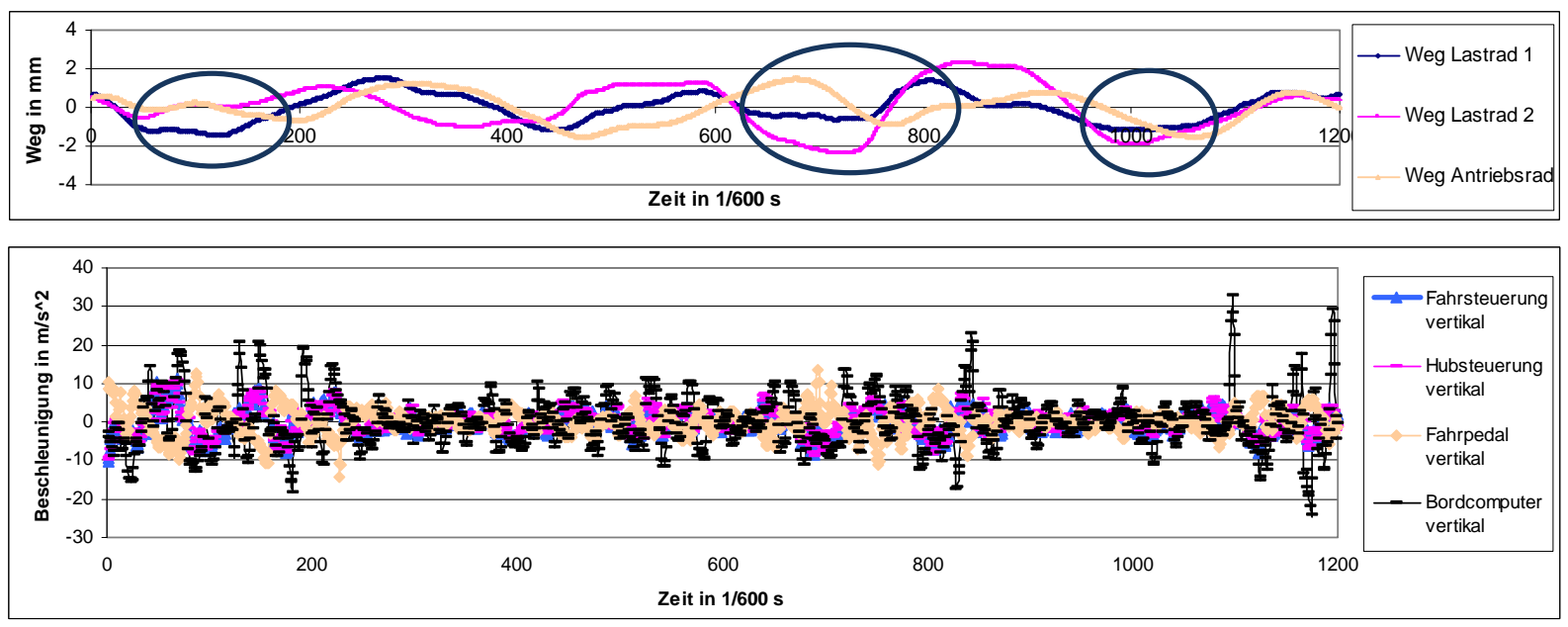

Abbildung 3: Weg- und Beschleunigungsverläufe von Fahrzeugteilen und der oben genannten Elektronikkomponenten

Im Rahmen von Zuverlässigkeitsuntersuchungen sind in erster Linie sehr hohe auftretende Beanspruchungen der Bauteile von Interesse. Daher wird am Beispiel von (c) 2010 Logistics Journal : Proceedings - ISSN 2192-9084 
Zeitabschnitten mit hohen Beschleunigungsamplituden das Zustandekommen von Höchstbelastungen analysiert. Wie aus Abbildung 3 hervorgeht, werden die höchsten Beschleunigungswerte von mehrfacher Erdbeschleunigung jeweils bei Zeitabschnitten mit verhältnismäßig großen Bewegungen der Teller von bis zu $4 \mathrm{~mm}$ in 0,1 Sekunden erreicht. Die größten vertikalen Beschleunigungen sind jeweils bei stark gegenläufigen Bewegungen der drei Teller des Hydropulser-Prüfstands zu beobachten. Diese Bereiche sind im Diagramm eingekreist dargestellt. Die Abbildung 4 zeigt, dass die vertikale Beschleunigung der Fahrsteuerung einen ähnlichen Verlauf aufweist wie die Lagerung der Antriebseinheit, in deren Nähe sie verbaut ist.

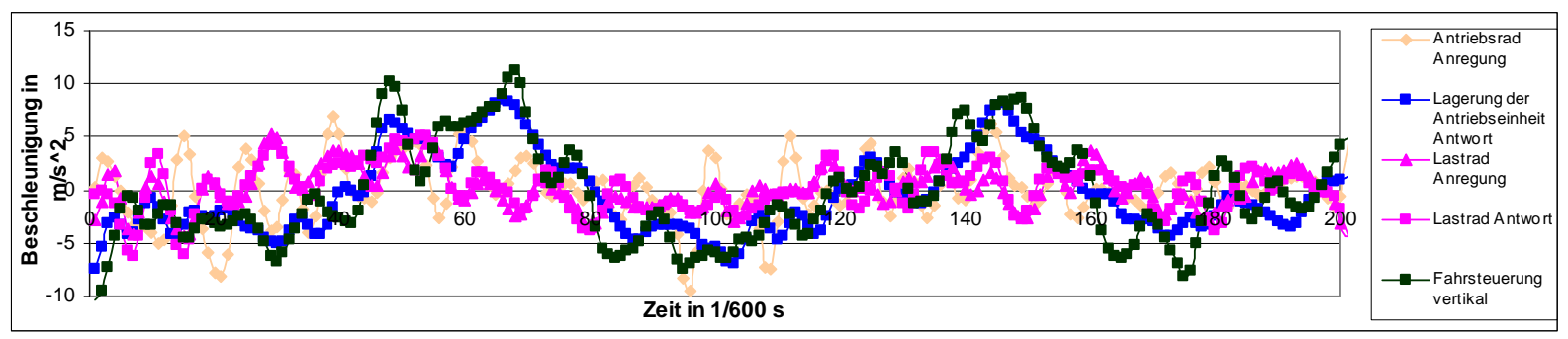

Abbildung 4: Beschleunigungsverläufe von Fahrzeugteilen und der Fahrsteuerung

Im oberen Teil wird die Untersuchung der Zeitverläufe von gemessenen Schwingungen beschrieben. Die Abbildung 5 zeigt das Ergebnis einer Kurzzeit-Diskreten Fourier-Transformation (Kurzzeit-DFT) bezüglich der Antwort der Lagerung der Antriebseinheit, eines Lastrades und eines Bedienelementes auf die Hydropulser-Anregung. Da diese Analyse zur Übertragung der Zeitverläufe in den Frequenzbereich nur für periodische Signale angewendet werden darf, wird zur Minimierung des Fehlers nur ein kurzes Zeitfenster betrachtet. Aus dem Leistungsspektrum geht hervor, dass insbesondere der niedere Frequenzbereich dicht besetzt ist. Dies deutet auf eine Anregung durch ein Rauschsignal beziehungsweise auf unregelmäßige Schwingungen hin. Diese beruhen insbesondere auf Stoßbelastungen, die an den Umkehrpunkten der drei Teller entstehen.

Bei Bewertung der DFT ist zu berücksichtigen, dass diese Analyse nicht zur Ermittlung von Eigenwerten geeignet ist, da die zufällige Aneinanderreihung von Frequenzen einer gezielten Anregung einzelner Frequenzen widerspricht. Auch lassen sich zumindest im Fall mehrfacher Stöße selbst für periodische Signale mithilfe einer DFT keine Eigenfrequenzen aufspüren [Zsc00]. Die Leistungsspektren lassen ebenfalls nur qualitative Aussagen hinsichtlich der Beschleunigungsamplituden zu, da für präzise Werte neben einer hohen Abtastfrequenz und großen Signallänge insbesondere aufgrund des Faltungsprozesses bei der DFT ein periodisches Signal benötigt wird [Hof05]. Es können dennoch einige Aussagen aus den vier Signalen aus Abbildung 5 abgeleitet werden. Die Querbeschleunigung des Bediengerätes beispielsweise weist in der Tat für das betrachtete Signal das größte Leistungsspektrum auf. Der relativ schmale Frequenzbereich folgt aus den gleichmäßigen Frequenzen des Eingangssignals. Im Gegensatz dazu wird bei Antwort des Lastrades und der vertikalen Beschleunigung des Bedienelements ein breites Frequenzspektrum angesprochen, da (c) 2010 Logistics Journal : Proceedings - ISSN 2192-9084 
die Frequenzen in dem kurzen betrachteten Signal ebenfalls insbesondere für das Bedienelement stark variieren. Zur genaueren Betrachtung der Beschleunigungsverläufe soll ergänzend die kontinuierliche Fourier-Transformation angewendet werden.

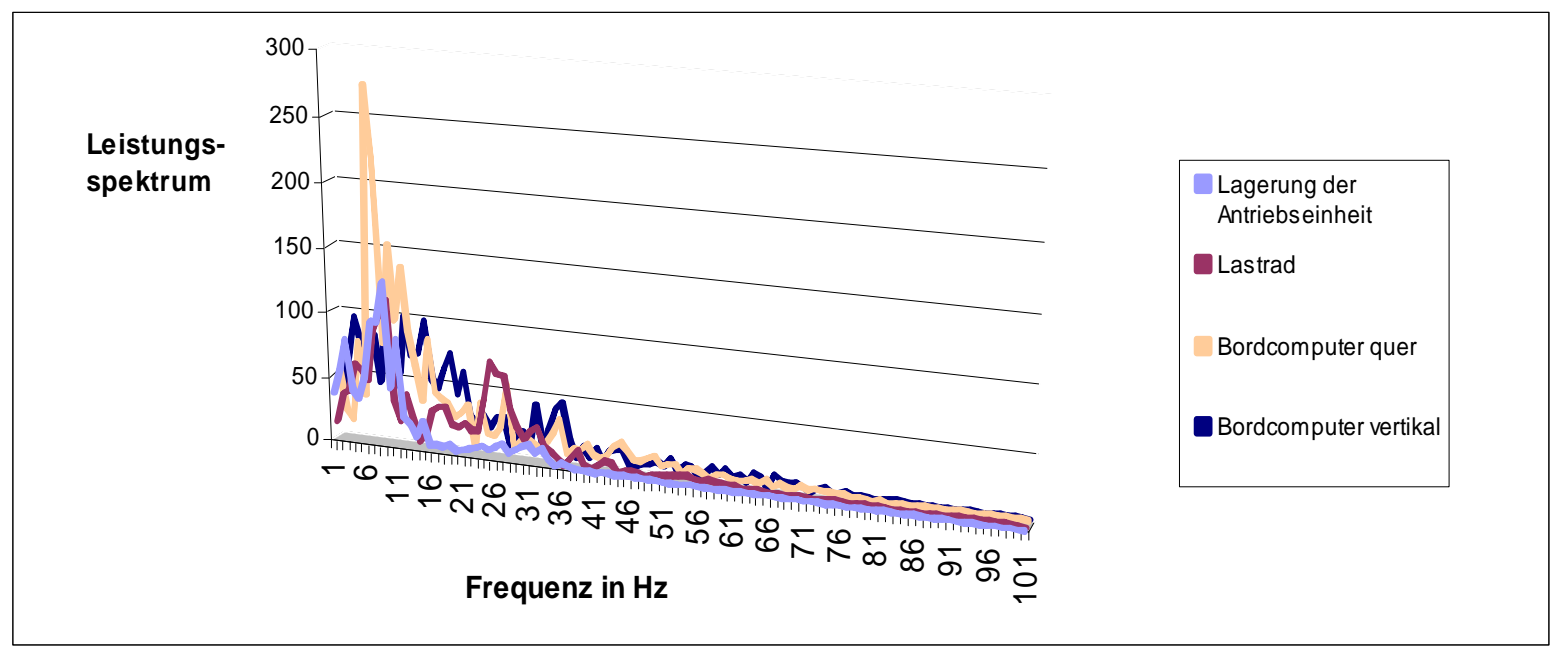

Abbildung 5: Kurzzeit-Diskrete Fourier-Transformation

\subsection{Ergebnisse der Auswertung}

Im Folgenden werden die Ergebnisse der Messdaten-Auswertung zusammengestellt:

- Bei Betrachtung der Beschleunigungswerte in y- und z-Richtung zeigt sich, dass die Bedienelemente in der Fahrzeugkabine höhere Werte aufweisen als die Steuerungen, die sich im unteren Teil des Fahrzeugrahmens befinden.

- Wie im Fahrbetrieb treten die maximalen Belastungen für beide Richtungen jeweils bei Überlagerung verschiedener Belastungsarten auf. Auf dem Hydropulser-Prüfstand werden diese durch gegenläufige Bewegung der Teller unter den Rädern hervorgerufen.

- Die gemessenen maximalen Querbelastungen liegen jeweils leicht unterhalb der maximalen vertikalen Beschleunigungen. Die höchsten Querschwingungen werden im Fahrbetrieb wie auch auf dem Hydropulser über die Räder eingeleitet. Die Ursachen für Querschwingungen bei Einsatz eines Schubmaststaplers bilden Schäden an den Rädern, eine Kurvenfahrt, außermittig angeordnete Lasten sowie vor allem Bodenunebenheiten und eine Überfahrt über eine versetzte Schwelle.

Für die geplanten Zuverlässigkeitstests auf einem elektrodynamischen Shaker dienen die ermittelten Höchst- und Durchschnittsbelastungen als Leitwerte für real auftretende mechanische Belastungen. Mithilfe dieser Beschleunigungssignale und von Vergrößerungsfaktoren sind ferner zeitraffende Alterungsbeständigkeitstests durchführbar. Ein Schwingprüfsystem kann zudem für experimentelle Modalanalysen bei den betrachteten Elektronikkomponenten eingesetzt werden. 


\section{Aufnahme der elektrischen Belastungen}

\subsection{Messwertaufnahme an einem Versuchsstapler}

Zur Aufnahme der elektrischen Belastungsprofile wurde am Institut für Transportund Automatisierungstechnik ein vorhandener Gegengewichtsstapler mit Sensoren und Messwertaufnehmern für die Strom- und Spannungsmessung ausgestattet. Da für die Ermittlung dieser Größen unterschiedliche Messmethoden und Aufnahmeparameter zu Verfügung stehen, wurden nach den ersten Probemessungen zunächst diejenigen Verfahren ausgewählt, welche die für das Zerstörungsszenario relevanten Einflüsse am genauesten abbilden. In diesem Zusammenhang wurden auch die zunächst für die Messung der auftretenden Ströme verwendeten Kompensationsstromwandler durch Nebenschlusswiderstände ersetzt, da diese Stromspitzen und einbrüche besser erkennbar machen und zudem über eine geringere Temperaturempfindlichkeit verfügen. Mit ihrem genau tolerierten Widerstandswert der Genauigkeitsklasse 0,5 nach DIN 43703 [DIN76] ermöglichen sie über den gemessenen Spannungsabfall die Bestimmung der Stromstärke mit Hilfe des ohmschen Gesetzes. Der Einbau der Nebenschlusswiderstände in die Zuleitungen der Motorsteuerungen erfolgte in einer geschlossenen Kunststoffbox direkt vor den Steuerungen um die Leitungswege möglichst kurz zu halten und das Kurzschlussrisiko zu minimieren (Abbildung 6: Verkabelung der Motorsteuerungen (rot) zur Aufnahme von Strom- und Spannungsprofilen).

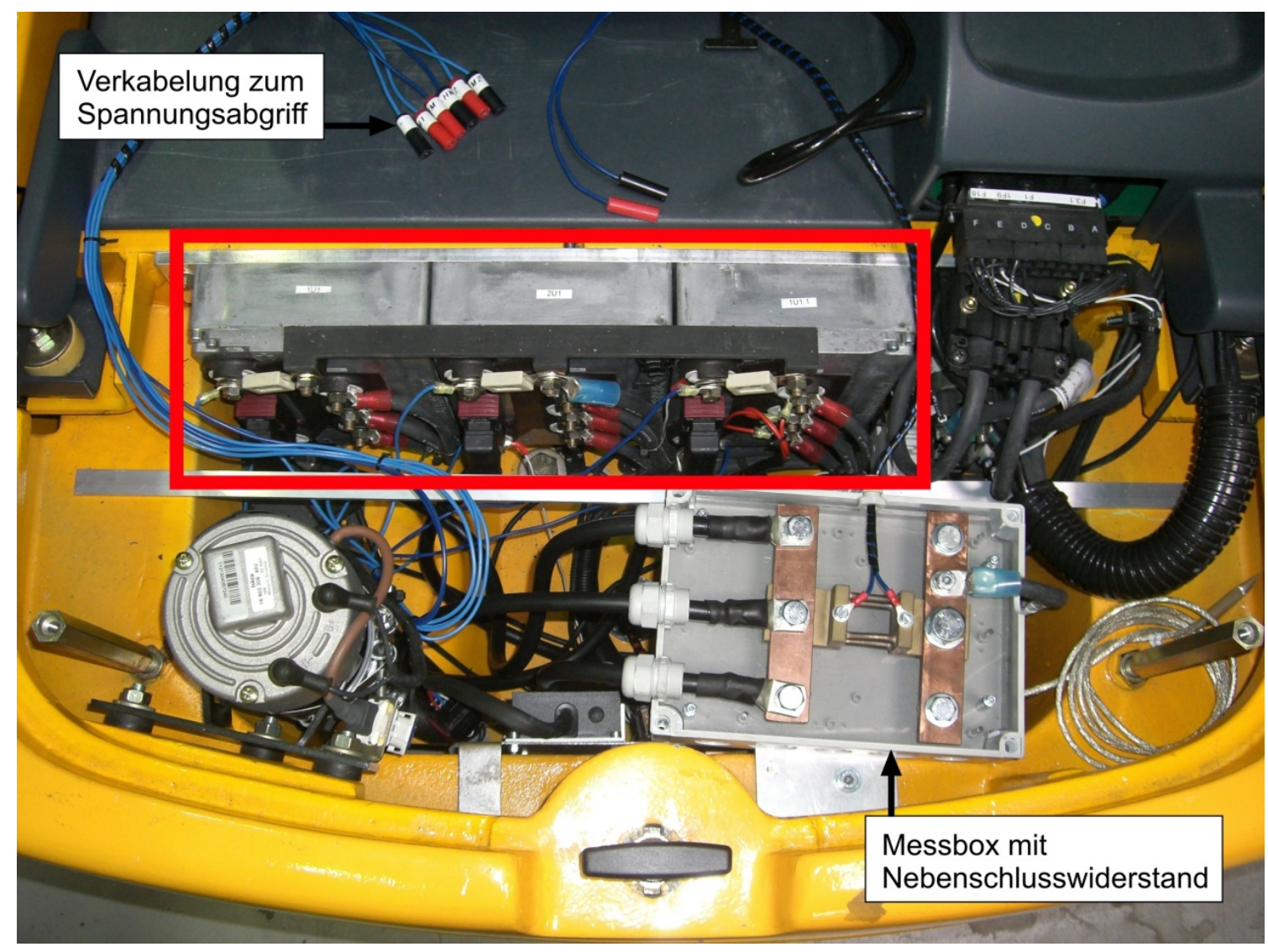

Abbildung 6: Verkabelung der Motorsteuerungen (rot) zur Aufnahme von Strom- und Spannungsprofilen 
Die Verarbeitung und Speicherung der ermittelten Strom- und Spannungswerte übernimmt ein EDV-System mit einem angeschlossenen Datenerfassungsgerät von National Instruments, dass über ein eingesetztes Analogeingangsmodul eine Summenabtastrate von bis zu 800.000 Werten pro Sekunde ermöglicht. Mit dieser hohen Messfrequenz sollen auch gegebenenfalls vorhandene Störimpulse mit sehr kurzen Anstiegszeiten und Impulslängen sichtbar gemacht werden. Derartige Einwirkungen werden im Flurförderzeugbereich oftmals durch elektrostatische Entladungen (ESD) hervorgerufen und können mirkoelektronische Schaltungen nachhaltig schädigen [CG03]. Um bei der Auswertung der aufgezeichneten Messdaten eine Zuordnung von aufgetretenen Störeffekten und auffälligen Signalabschnitten bestimmten Fahrzuständen und Bedienmanövern zuordnen zu können, wurde ein Dualkamerasystem des Herstellers Mobotix in den Messaufbau integiert. Die beiden Bildsensoren überwachen dabei den Fahrerplatz mit sämtlichen Bedienungseinrichtungen und das Fahrersichtfeld vor dem Stapler. Die über eine Ethernet-Schnittstelle an das EDVSystem übertragenen Videodaten werden ebenso wie die Signalverlaufsgraphen der momentanen Messgrößen in einer mit LabVIEW programmierten Benutzeroberfläche angezeigt. Mit Hilfe einer Recordingsoftware kann zudem das komplette Bildschirmsignal aufgezeichnet und gesichert werden (Abbildung 7: Benutzeroberfläche mit eingebundenem Kamerasystem während der Datenaufzeichnung).

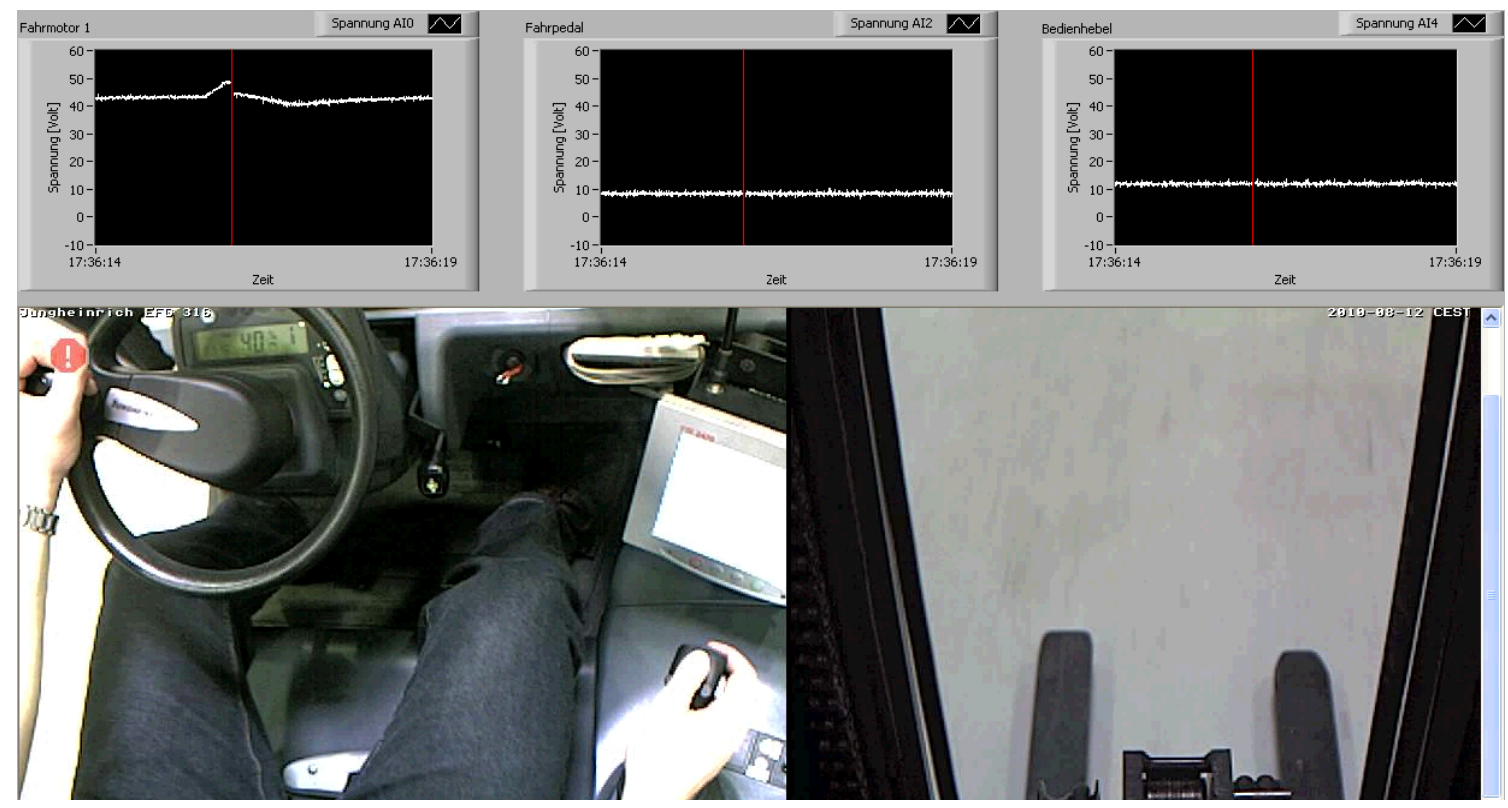

Abbildung 7: Benutzeroberfläche mit eingebundenem Kamerasystem während der Datenaufzeichnung

Die Energieversorgung der Messelektronik und des EDV-Systems stellt eine separate Gel-Batterie mit angeschlossenem Spannungswandler sicher, die mit weiteren Komponenten hinter dem Fahrersitz des Staplers montiert wurde. Der elektrisch von der Fahrzeugelektronik getrennte Aufbau soll dabei mögliche Einflüsse auf das zu analysierende Fahrzeugsystem vermeiden.

Für die Versuchsfahrten zur Aufzeichnung der elektrischen Belastungsprofile wurden auf Basis von sechs vorhandenen Nutzungsprofilen von Gabelstaplerbetreibern unter 
Variation verschiedener Parameter, wie beispielsweise der Nutzlast oder der Hubhöhe, unterschiedliche praxisnahe Arbeitsspiele definiert. Hierbei ist auch ein bereits veröffentlichtes Modell zur Simulation des Fahrweges [Sch06], als auch die in der DIN EN 13059 [EN09] vorgeschlagene Versuchsstrecke zur Feststellung von Schwingungsbelastungen auf den Fahrer, berücksichtigt worden.

\subsection{Ergebnisse der Aufnahme elektrischer Messgrößen}

Um festgestellte Auffälligkeiten in den aufgezeichneten Signalverläufen reproduzierbar zu bestimmten Einsatzbedingungen zuordnen zu können, wurden die Messungen mit den aufgestellten Arbeitsspiele und ihren festgelegten Variationen jeweils in mehrfacher Anzahl absolviert. In Bezug auf die Versorgungsspannungen zeigte sich bei der Auswertung der aufgezeichneten Daten, dass diese an einzelnen Komponenten je nach Fahrsituation des Staplers kurzzeitig um bis zu 20\% ansteigen oder einbrechen können. Hiervon sind insbesondere die Fahr- und Hubmotorsteuerungen betroffen, die als Komponenten der Leistungselektronik direkt von der Traktionsbatterie mit einer Nennspannung von $48 \mathrm{~V}$ gespeist werden. Desweiteren konnte bei der Variation der Messfrequenz an den Gleichspannungseingängen der Motorsteuerungen der Trend zu weiter auseinanderliegenden Extremwerten bei einer Erhöhung der Abtastrate beobachtet werden (Abbildung 8: Auswertung von aufgezeichneten Spannungsverläufen mit unterschiedlicher Messfrequenz).

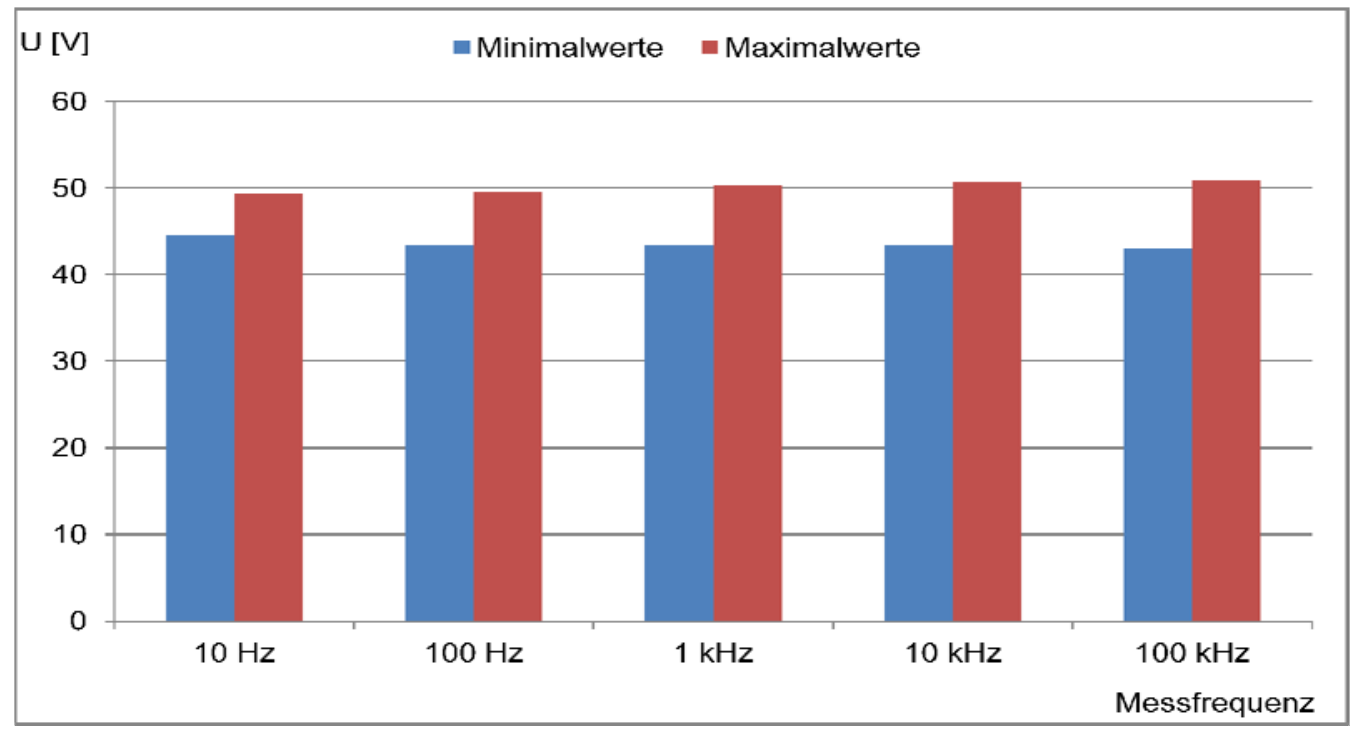

Abbildung 8: Auswertung von aufgezeichneten Spannungsverläufen mit unterschiedlicher Messfrequenz

Wie die weitere Analyse der Messgrößenverläufe ergab, ist dieses einer Überlagerung des Grundsignals durch hochfrequente Anteile geschuldet. Bei der Identifikation der zu starken globalen Spannungsspitzen bzw. -einbrüchen führenden Betriebssituationen zeigte sich mit Hilfe der aufgezeichneten Videodaten, dass insbesondere das Aufwecken des Gabelstaplers aus dem Standby-Modus sowie ein schneller Richtungswechsel durch Betätigung des Fahrtrichtungsschalters bei Maximalge- 
schwindigkeit zu derartigen Effekten führt (Abbildung 9: Versorgungsspannung der Fahrmotorsteuerung bei doppeltem schnellen Richtungswechsel).

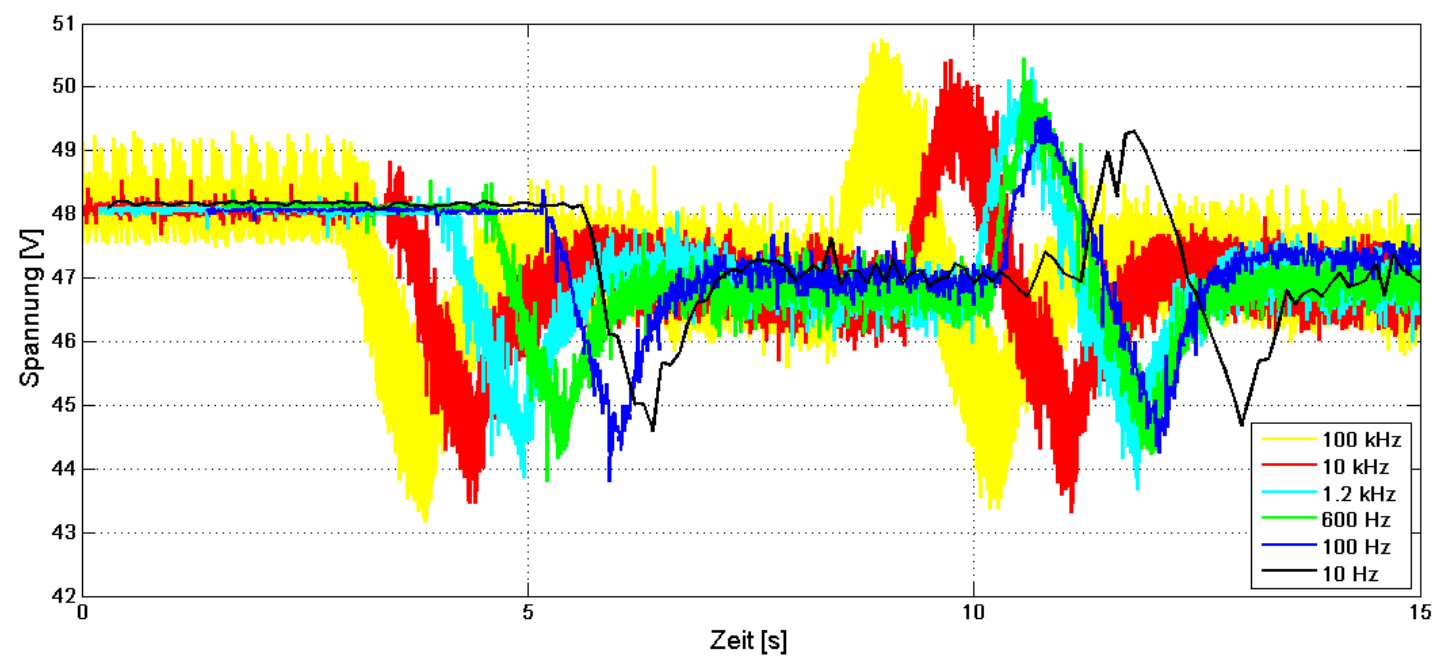

Abbildung 9: Versorgungsspannung der Fahrmotorsteuerung bei doppeltem schnellen Richtungswechsel

Als Ergebnis der Aufnahme elektrischer Belastungen stehen Strom- und Spannungsverläufe zu unterschiedlichen und in der Praxis häufig anzutreffenden Arbeitsspielen zur Verfügung. Durch das in Abschnitt 3.1 vorgestellte Kamerasystem ist es dabei auch nachträglich möglich, charakteristische Abschnitte in den Messgrößenverläufen bestimmten Betriebszuständen und Fahrmanövern zuzuordnen (Abbildung 10: Stromprofil an einer Fahrmotorsteuerung während verschiedener Fahrzustände; Abbildung 11: Stromprofil an einer Fahrmotorsteuerung während des Einlagerns einer Palette).

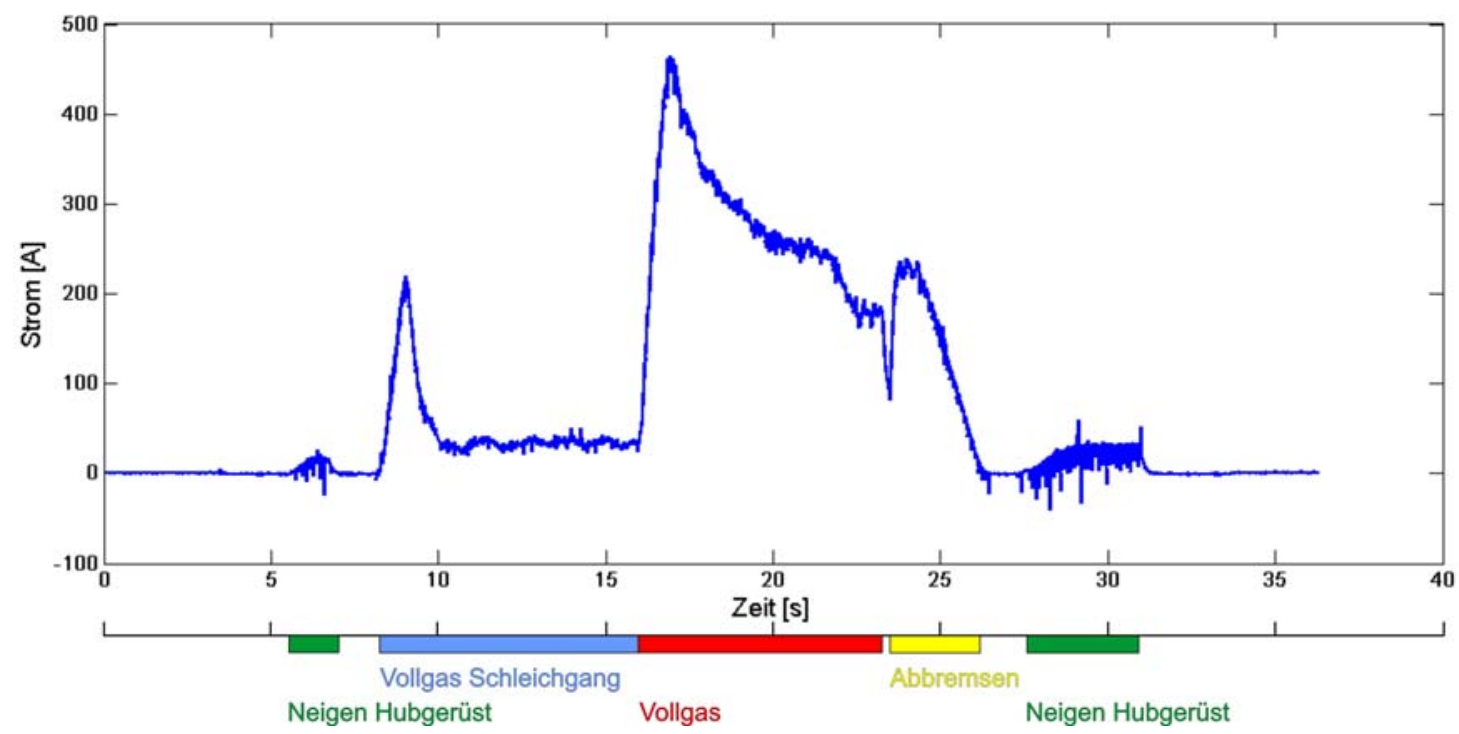

Abbildung 10: Stromprofil an einer Fahrmotorsteuerung während verschiedener Fahrzustände 


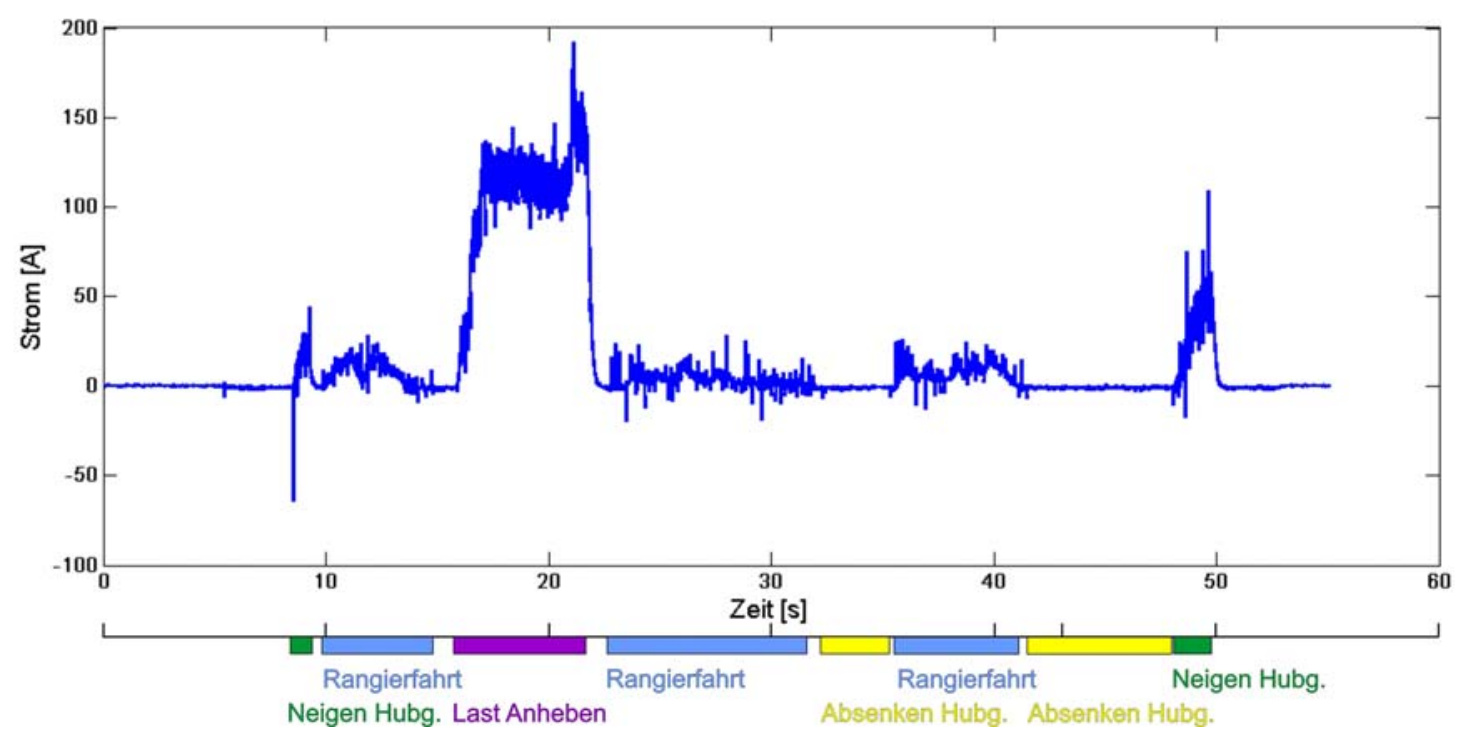

Abbildung 11: Stromprofil an einer Fahrmotorsteuerung während des Einlagerns einer Palette

Weitere Untersuchungen unter Variation von Fahrbahnbelägen, Ladezuständen der Traktionsbatterie sowie der Umgebungstemperatur werden bis zum Ende des Jahres die bisher durchgeführten Versuchsreihen und zusammengestellten elektrischen Belastungskollektive ergänzen.

Aneinandergereiht spiegeln die aufgenommenen Strom- und Spannungsverläufe typische Belastungen elektronischer Komponenten im alltäglichen Betrieb eines Flurförderzeuges in konzentrierter Form wieder. Als Zwischenergebnis des Forschungsprojekts stehen sie für die Produktion und Entwicklung von Elektronikkomponenten in Flurförderzeugen bereits zur Verfügung.

In den nächsten Projektschritten dienen die praxisnahen Beanspruchungsprofile als Basis für Reihenmessungen an einzelnen Komponenten. Die Simulation dieser elektrischen Belastungen erfolgt dabei in Bezug auf die Fahr- und Hubmotorsteuerungen an einem Motorenprüfstand. Alle weiteren ausgewählten Komponenten werden mit einem programmierbaren Netzgerät den aufgezeichneten und teils stark schwankenden Spannungsverläufen ausgesetzt. An beiden Komponentengruppen werden zudem mit einem ESD-Generator Luft- und Kontaktentladungen von bis zu $30 \mathrm{kV}$ simuliert, die in der Praxis häufig zu Störungen und Ausfällen von elektronischen Komponenten in Flurförderzeugen führen. 


\section{$4 \quad$ Ausblick}

Die aus den Messdaten abgeleiteten elektro-mechanischen Belastungskollektive bilden im weiteren Verlauf des Forschungsprojekts die Basis für Belastungs- und Zuverlässigkeitstests unter Laborbedingungen.

Hierbei werden zudem die Kombination von mechanischen und elektrischen Belastungen sowie der thermische Einfluss in Hinblick auf mögliche Wechselwirkungen der einzelnen Belastungsarten untereinander untersucht.

Aus der Analyse der defekten beziehungsweise vorgeschädigten Komponenten werden Modelle zur Darstellung des Zusammenhangs zwischen Belastungen und Ausfällen erarbeitet und Empfehlungen zu bestimmten Technologien sowie qualitative und quantitative Dimensionierungskriterien für elektronische Komponenten in Flurförderzeugen abgeleitet.

\section{Literatur}

[Bay10] Bayer MaterialScience: Mit Vulkollan ${ }^{\circledR}$ ist Ihnen der Erfolg sicher! Leverkusen: Bayer MaterialScience, Cast Elastomers, 2010.

[Bei94] Beisteiner, Franz: Stapler: Beanspruchungen, Betriebs-verhalten und Einsatz. Renningen: Expert-Verlag, 1994.

[CG03] Camp, Michael; Garbe, Heyno: Wenn der Chip streikt - Der Ausfall mikroelektronischer Schaltungen kann weit reichende Konsequenzen haben, Hannover: UNI-Magazin, 1/2-2003.

[DIN76] DIN Deutsches Institut für Normung e.V.: DIN 43703: Elektrische Messgeräte - Nebenwiderstände. Berlin: Beuth Verlag, 1976.

[EN09] DIN Deutsches Institut für Normung e.V.: Deutsche Fassung EN 13059: Sicherheit von Flurförderzeugen - Schwingungsmessung. Berlin: Beuth Verlag, 2009.

[Hof05] Hoffmann, Rüdiger: Grundlagen der Frequenzanalyse, Band 2. Renningen: Expert-Verlag, 2005.

[VDI10a] VDI-Gesellschaft Produktion und Logistik: VDI 2695: Ermittlung der Betriebskosten für Diesel- und Elektro-Gabelstapler. Berlin: Beuth Verlag, 2010.

[VDI10b] VDI-Gesellschaft Produktion und Logistik: VDI 4461: Beanspruchungskategorien für Gabelstapler. Berlin: Beuth Verlag, 2010

[Sch06] Schmalzl, Jürgen: Simulation des dynamischen Verhaltens von Flurförderzeugen der Lagertechnik. München: Utz Verlag, 2006.

[Zsc00] Zschieschang, Torsten: Schwingungsanalyse an Maschinen mit ungleichförmig übersetzenden Getrieben. Chemnitz: Dissertation, 2000. 